\title{
An integrated in vitro model of perfused tumor and cardiac tissue
}

\author{
Monica Moya ${ }^{\dagger 1,2}$, David Tran ${ }^{\dagger 2,3}$ and Steven C George ${ }^{* 1,2,3,4}$
}

\begin{abstract}
Cancer and cardiovascular disease remain the two leading causes of death in the United States. Progress in treatment to reduce morbidity and mortality will include the development of new drugs. Recent advances in induced pluripotent stem cell technology, tissue engineering, and microfabrication techniques have created a unique opportunity to develop three-dimensional (3D) microphysiological systems that more accurately reflect in vivo human biology when compared with two-dimensional flat systems or animal models. Our group is working to develop 3D microphysiological systems using induced pluripotent stem cell technology that simulates the microcirculation, the cardiac muscle, and the solid tumor, and then to combine these systems into an integrated microphysiological system that simulates perfused cardiac muscle and solid tumor on a single platform. The platform will be initially validated to predict anti-cancer efficacy while minimizing cardiac muscle toxicity. A critical feature will be blood flow through a human microcirculation (capillaries and larger microvessels), which is necessary to overcome diffusion limitations of nutrients and waste products in realistic 3D cultures, and serves to integrate multiple organ systems. This is a necessary and critical feature of any platform that seeks to simulate integrated human organ systems. The results of our project should produce a new paradigm for efficient and accurate drug and toxicity screening, initially for anti-cancer drugs with minimal cardiac side effects, and a platform technology that can be eventually used to integrate multiple major organ systems of the human body.
\end{abstract}

Keywords: cardiomyocyte, microcirculation, induced pluripotent stem cell, microphysiological system, cancer

\section{Introduction}

Cancer and heart disease are, by far, the two leading causes of mortality in the United States. There remains a great need for continued innovation in the development of new therapeutic options including pharmaceutics. While two-dimensional (2D) cell culture systems remain the mainstay for drug screening, these systems do not capture the rich cell-cell and cell-matrix interactions of the three-dimensional (3D) in vivo tissue microenvironment [1,2]. Moreover, while there is not normally an obvious connection (for example, cardiac tumors) between the diseases, it is well documented that several prominent chemotherapeutic agents (for example, doxorubicin, cyclophosphamide) have detrimental effects on cardiac function [3]. In addition, both cardiomyocytes and many tumor cells share expression of prosurvival

\section{${ }^{\dagger}$ Contributed equally}

*Correspondence: scgeorge@uci.edu

'Department of Biomedical Engineering, 2420 Engineering Hall, University of

California, Irvine, Irvine, CA 92697-2730, USA

Full list of author information is available at the end of the article proteins and tyrosine kinases, the latter of which is a promising new target for a family of anti-cancer agents [4].

To replicate the complex 3D arrangement of cells and extracellular matrix (ECM), new human microphysiological systems should include a physiologically relevant vascular supply, which not only provides the necessary convective transport of nutrients and waste, but also couples and integrates the response of multiple organ systems. The goal of our project is to create advanced 3D human microphysiological systems utilizing induced pluripotent stem cell (iPSC) technology that include the vasculature (Figure 1a), the cardiac muscle (Figure 1b), and the tumor (Figure 1c), in a flexible, modular, and integrated design (Figure 1d,e).

\section{Perfused network of microvessels}

Capillary networks are universal to all organ systems, including the heart, and can be affected by drugs that directly target the endothelial cell (EC) or target growth factors that stimulate angiogenesis such as vascular endothelial growth factor. In vivo, oxygen, nutrients, and waste products are transported through the vascular 
(a)

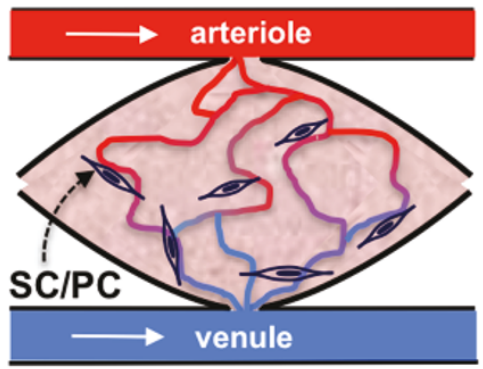

(b)

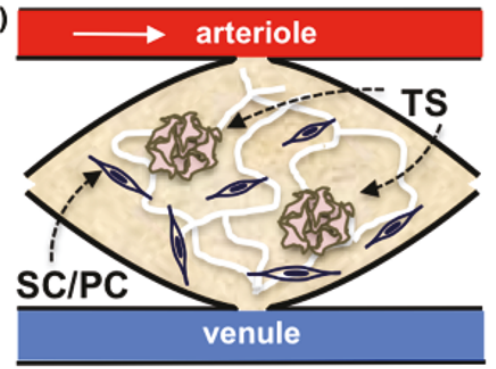

(c)

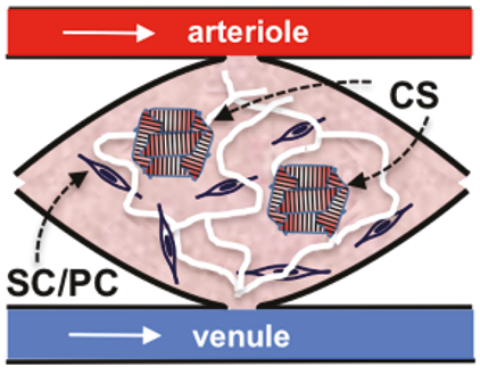

(d)

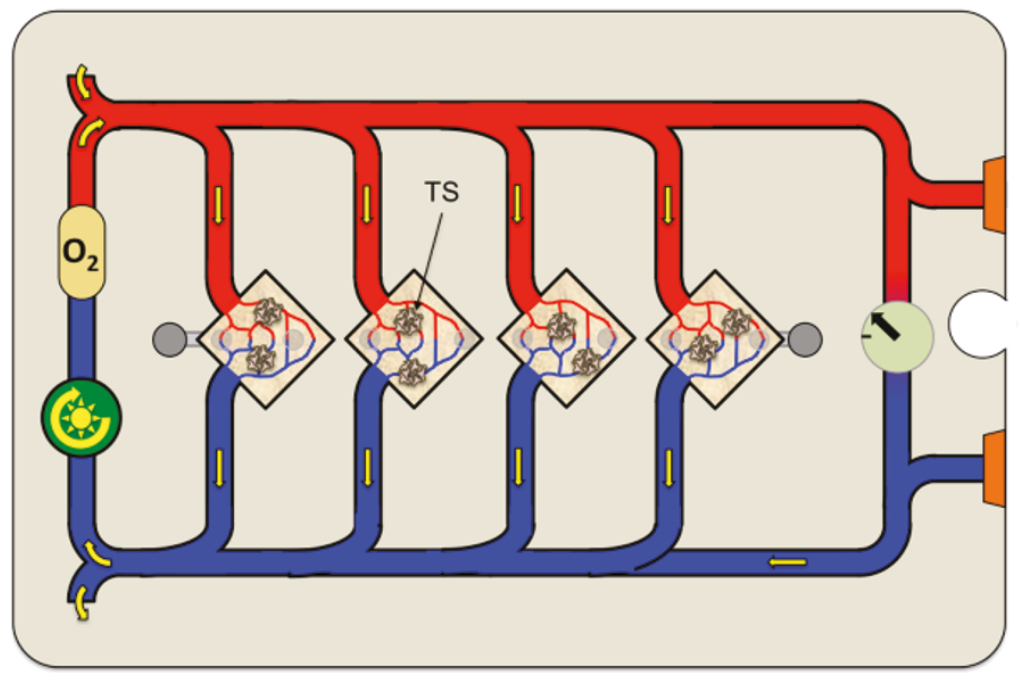

(e)

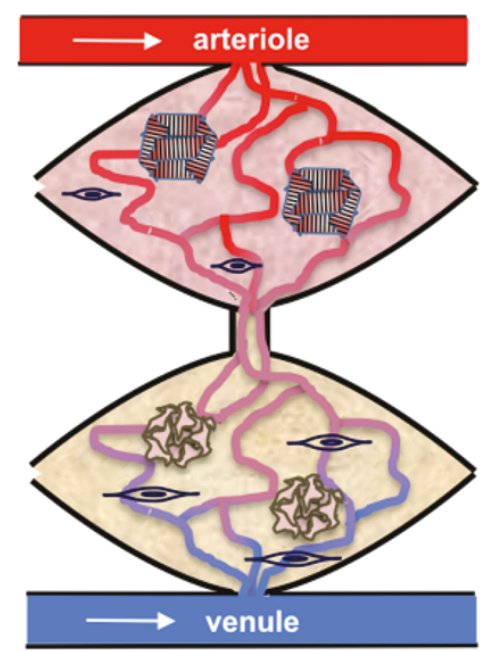

Figure 1. The project will create three separate microphysiological systems that can then be incorporated. Three separate microphysiological systems will simulate: (a) a perfused network of human capillaries derived from human induced pluripotent stem cells in a porcine cardiac extracellular matrix, (b) a network of human capillaries in a the presence of cardiac muscle spheroids derived from the human induced pluripotent stem cells, and (c) a network of human capillaries in the presence of solid tumor spheroids. As depicted, the arteriole and venule are microfluidic channels of high pressure/high oxygen and low pressure/low oxygen, respectively. (d) Any of these individual microphysiological systems can be incorporated into a modular high-throughput platform that includes a row of perfused microphysiological systems (perfused tumor spheroids shown as an example). The platform can include modular connectors to facilitate addition of other organ systems. (e) The final phase is to combine the individual microphysiological systems into a single integrated system of perfused tumor spheroid and cardiac muscle. SC, stromal cells; PC, pericyte-like cell; TS, tumor spheroid; CS, cardiomyocyte spheroid.

network by convection, or the bulk movement of fluid to meet the dynamic and complex needs of metabolic tissues. Current models to investigate the microcirculation include human studies that are costly and have limited potential for mechanistic intervention, in vivo animal studies that require extrapolation to human biology, or static in vitro models that employ purified one-component ECM materials (for example, collagen, fibrin), which do not adequately reflect the diversity of ECM composition between tissues.

Developing dynamic 3D vasculature-fed organ-specific in vitro human microtissues has the potential to provide whole new opportunities for discovery while reducing the use of animals in research. Current in vitro models that lack interstitial flow and a perfused capillary network offer a limited mimicry of the tissue microenvironment. ECs experience fluid shear forces throughout the vascular tree that can impact their function. Interstitial flow not only provides important biomechanical cues in the microenvironment, but can also markedly impact extracellular gradients of solutes or small molecules. Additionally, the microcirculation is the major conduit for drug delivery to tissues.

Recently, microfabrication technology has led to the creation of precise microchannels on nonbiological substrates (for example, silicon or polydimethylsiloxane) [5] or within purified single-component substrates such as collagen [6]. While these approaches introduce convection as a mechanism of transport, even when endothelialized, the channels are not human capillaries and thus lack the flexibility to adapt to changing metabolic needs.

To create patient-specific vascular networks, our group will expand our previous work in engineering vasculature [7-9] in vitro by developing vessel networks derived from 
human iPSC-derived ECs in a cardiac-derived ECM. Over the past 2 years, we have developed a novel microfluidic-based system that supports a metabolically active stroma with culture medium-perfused human capillaries [10]. In the original iteration of the design, endothelial colony-forming cell (ECFC)-derived ECs harvested from cord blood were used to form vessels with the support of stromal cells, normal human lung fibroblasts, in a fibrin matrix. More recently, we demonstrated the versatility of this platform to form robust capillary networks using various matrices such as type I collagen and porcine cardiac-derived ECM [11] blended with fibrin (Figure 2a). Using ECFC-derived ECs, we achieved perfused microvessels in the cardiac-derived ECM blend (Figure 2b). Current work is focused on creating a patient-specific 3D perfused vascular network using human iPSC-derived ECs.

\section{Perfused cardiac muscle}

Accurately predicting adverse cardiac side effects of new pharmaceutical drugs currently relies heavily on animal models or $2 \mathrm{D}$ and relatively simple $3 \mathrm{D}$ in vitro models $[12,13]$. Although animal models allow insight into pharmacokinetics and whole organ drug response, some drugs have been shown to only effect cardiomyocytes of human origin [14]. This has lead to unexpected and undesirable cardiotoxicity in human clinical trials that were not predicted in preclinical animal testing. Alternatively, human iPSC-derived cardiomyocytes offer many advantages over animal models, including human origin, culture adaptation, and ability to create patientspecific cell lines. Furthermore, human iPSC-derived cardiomyocyte 2D monolayers exhibit predictable responses to known cardioactive drugs $[15,16]$. Nonetheless, in order to fully mimic the human response, human iPSC-derived cardiomyocyte drug screening platforms should be multicellular (for example, contain cardiomyocytes, stromal cells, ECs) [17], should be 3D [18,19], and should have nutrients and drugs delivered physiologically through the microcirculation.

We demonstrate feasibility for using our proposed system to create a vascularized cardiac microtissue by first differentiating human iPSCs (generous gift from Professor Bruce Conklin, Gladstone Institutes, San Francisco, CA, USA) into cardiomyocytes following a matrix sandwich method [20]. Briefly, human iPSCs are cultured as a monolayer on matrigel and then overlaid with matrigel while sequentially exposed to activin $\mathrm{A}$ and bone morphogenic protein-4. Then 3D contracting cardiac organoids with physiologically relevant cell density of $10^{8}$ to $10^{9}$ cells $/ \mathrm{cm}^{3}$ are formed using AggreWell ${ }^{\mathrm{Ts}}$ plates (STEMCELL Technologies Inc., Vancouver, BC, Canada). We then combine the 3D cardiac organoids with ECFCderived ECs and normal human lung fibroblasts in a microfluidic platform [10]. Human iPSC-derived cardiomyocytes survive and continue to contract within the device for up to 28 days while a surrounding vessel network develops (Figure 2c).

Our polydimethylsiloxane-based microdevice is transparent, enabling the use of non-invasive and nondestructive optical techniques to probe and characterize cardiomyocyte function. Changes in beat frequency and force are tracked using brightfield microscopy while the electrophysiology of cardiomyocytes is visualized using voltage-sensitive dyes. Drug-induced cardiotoxicity can be monitored using the terminal deoxynucleotidyl transferase dUTP nick end-labeling assay. Finally, the metabolism will be tracked by measuring the ratio of protein bound:free $\mathrm{NADH}$ using fluorescent lifetime imaging [21].

Future research will focus on creating capillary perfusion within our 3D model of vascularized cardiac tissue and then validating the cardiac response with a panel of drugs with known mechanism (for example, epinephrine). Current $3 \mathrm{D}$ in vitro models have yet to incorporate vasculature necessary for physiological convective transport of nutrients, waste removal, and drug delivery to human iPSC-derived cardiac tissue - or any other functional human tissue, for that matter.

\section{Perfused solid tumor}

The study of the tumor microenvironment relies heavily on animal models $[22,23]$ or $2 \mathrm{D}$ and relatively simple 3D in vitro models [24-26]. In addition, multicellular tumor spheroids have been employed, but do not feature a perfused capillary network [27-29]. Animal models are capable of simulating the aggregate response of the tumor and host, but suffer from limitations in the response of a species, and are severely limited in their ability to screen large libraries of potential anti-cancer drugs. In vitro models have focused on the response of isolated tumor cells to a soluble factor(s) [30] or neighboring cells (for example, fibroblasts) [31]. These models have provided a wealth of information regarding intercellular signaling pathways in tumor cells. Unfortunately, most tumor cells are programmed with redundant and dynamically changing pathways that control differentiation and migration, and also respond to multiple factors within the microenvironment. Finally, it is unrealistic to create 3D models of all NCI60 tumor cell lines; hence, because anti-colon cancer drugs have known cardiac toxicity, our focus is on two colon cancer cells lines that are part of the NCI60 (SW620 and HCT116). This focus provides the opportunity to compare the response of our 3D system with a large body of data collected in simpler 2D systems, while also focusing our efforts on a model system that has the potential to distinguish the efficacy of new chemotherapeutic agents 

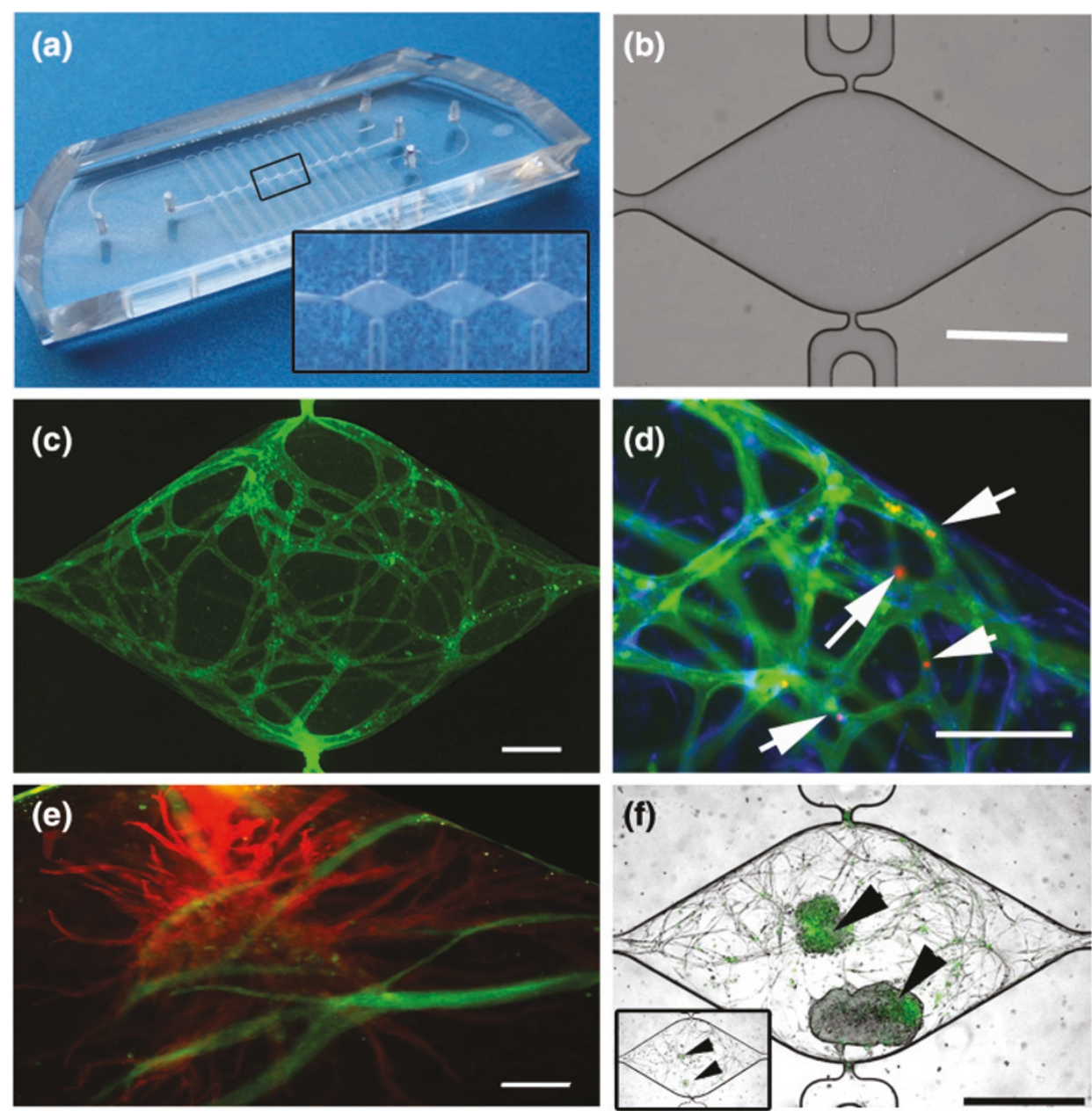

Figure 2. The microfluidic platform is conducive to the development of human microvessel networks. (a) Perfusable three-dimensional microvessels are generated using an optically clear polydimethsiloxane microfluidic-based platform, (b) consisting of two fluid-filled microfluidic channels on either side of $12 \mathrm{~mm}$ diamond-shaped tissue microchambers. Scale bar $=500 \mu \mathrm{m}$. The fluidic channels loop down and connect with each diamond through a single $30 \mu \mathrm{m}$ diameter pore that represents the only port for transport of nutrients and waste. A coculture of endothelial colony-forming-derived endothelial cells and normal human lung fibroblasts are mixed with fibrin matrix or another blend of extracellular matrix and microinjected into the central tissue chamber and allowed to gel. By 14 to 21 days, a robust network of microvessels develops. (c) Fluorescent microscopy of CD31-stained (green) microtissues at 18 days depicts an interconnected network of vessel in a porcine cardiac-derived extracellular matrix blend. Scale bar = $200 \mu \mathrm{m}$. (d) Vessel patency and perfusion is verified by introducing microspheres (red, white arrows) into the fluidic channels and observing their movement through the network. Scale bar $=200 \mu \mathrm{m}$. (e) A third cell type, such as tumor or cardiomyocyte spheroids, can also be added to the tissue chamber to create specific microorgan systems. Cardiomyocyte spheroids (cTnT, red) remain viable over 29 days in the microfluidic device as the vessel network (CD31, green) develops in the surrounding tissue. Scale bar $=100 \mu \mathrm{m}$. (f) Tumor spheroids (black arrows) from colorectal cancer cell line SW620 (transduced with Wnt-regulated GFP reporter cassette) proliferate and increase significantly in total mass at the same time as the continuous vessel network develops, especially between day 10 (inset) and day 20. Scale bar $=500 \mu \mathrm{m}$.

on epithelial-mesenchymal transition and early metastatic events. Our premise is that improved 3D models of the tumor microenvironment will significantly improve the efficiency of anti-cancer drug screening.

Our initial experiments demonstrate that our proposed model is appropriate to develop as a platform for the 3D tumor microenvironment. SW620 colorectal cancer cells, transduced with a Wnt-regulated GFP reporter cassette (generous gift from Professor Marian Waterman, University of California, Irvine, CA, USA), demonstrate significant growth between days 10 and 20 within the polydimethylsiloxane microdevice in the presence of fibroblasts and ECFC-derived ECs (Figure 2f). In addition, a vessel network develops rapidly over the course of the initial 10 days (Figure 2f).

A critical barrier to developing new anti-cancer drugs is the creation of a realistic in vitro model of the tumor microenvironment that has the potential to simulate key features such as the leaky and tortuous microcirculation. These features of the tumor microcirculation probably 
play an important role in early metastatic events such as intravasation. Our optically clear platform is ideal to view these events with high spatial and temporal resolution. The next steps in the development of the perfused solid tumor will be creating an environment in which rapid and reproducible anastomosis between the vessel network and the microfluidic channels occurs, and validating the response of the system to a panel of wellcharacterized anti-tumor drugs.

\section{Conclusion}

Cardiovascular disease and cancer remain the two leading causes of death in the United States, and innovative solutions to create new therapeutic interventions are needed. Our laboratory has spent the past decade developing 3D microphysiological systems [710,32-36], including recent results that demonstrate perfusion of living, dynamic human microvessels [10]. Our group is thus poised to develop microphysiological systems of cardiac muscle and solid tumor perfused by a living dynamic microcirculation on a single integrated platform. The results should produce a new paradigm for efficient and accurate drug and toxicity screening.

\section{Abbreviations}

2D, two-dimensional; 3D, three-dimensional; EC, endothelial cell; ECFC, endothelial colony-forming cell; ECM, extracellular matrix; iPSC, induced pluripotent stem cell.

\section{Competing interests}

The authors declare they have no competing interests.

\section{Author contributions}

MM, DT, and SCG all wrote specific sections of the manuscript, created the figures, and were involved in the final editing.

\section{Acknowledgements}

This work and the publication costs for this article were supported by grants from the National Institutes of Health (UH2 TR000481 and F32 HL105055).

\section{Declarations}

Publication of this supplement has not been supported by sponsorship. Articles have undergone the journal's standard review process. The Editors declare that they have no competing interests.

This article has been published as part of Stem Cell Research \& Therapy Volume 4 Supplement 1, 2013: Stem cells on bioengineered microphysiological platforms for disease modeling and drug testing. The full contents of the supplement are available online at http://www.stemcellres.com/supplements/4/S1.

\section{Author details}

'Department of Biomedical Engineering, 2420 Engineering Hall, University of California, Irvine, Irvine, CA 92697-2730, USA. ${ }^{2}$ Edwards Lifesciences Center for Advanced Cardiovascular Technology, University of California, Irvine, CA 92697, USA. ${ }^{3}$ Department of Chemical Engineering and Materials Science, University of California, Irvine, CA 92697, USA. ${ }^{4}$ Department of Medicine, University of California, Irvine, CA 92697, USA.

Published: 20 December 2013

\section{References}

1. Griffith $L G$, Swartz MA: Capturing complex 3D tissue physiology in vitro. Nat Rev Mol Cell Biol 2006, 7:211-224.
2. Roskelley CD, Desprez PY, Bissell MJ: Extracellular matrix-dependent tissuespecific gene expression in mammary epithelial cells requires both physical and biochemical signal transduction. Proc Natl Acad Sci U S A 1994, 91:12378-12382

3. Aiken MJ, Suhag V, Garcia CA, Acio E, Moreau S, Priebat DA, Chennupati SP, Van Nostrand D: Doxorubicin-induced cardiac toxicity and cardiac rest gated blood pool imaging. Clin Nucl Med 2009, 34:762-767.

4. Hasinoff BB: The cardiotoxicity and myocyte damage caused by small molecule anticancer tyrosine kinase inhibitors is correlated with lack of target specificity. Toxicol Appl Pharmacol 2010, 244:190-195.

5. Shin M, Matsuda K, Ishii O, Terai H, Kaazempur-Mofrad M, Borenstein J, Detmar M, Vacanti JP: Endothelialized networks with a vascular geometry in microfabricated poly(dimethyl siloxane). Biomed Microdevices 2004, 6:269-278.

6. Chrobak KM, Potter DR, Tien J: Formation of perfused, functional microvascular tubes in vitro. Microvasc Res 2006, 71:185-196.

7. Chen X, Aledia AS, Ghajar CM, Griffith CK, Putnam AJ, Hughes CC, George SC: Prevascularization of a fibrin-based tissue construct accelerates the formation of functional anastomosis with host vasculature. Tissue Eng Part A 2009, 15:1363-1371.

8. Chen X, Aledia AS, Popson SA, Him L, Hughes CC, George SC: Rapid anastomosis of endothelial progenitor cell-derived vessels with host vasculature is promoted by a high density of cotransplanted fibroblasts. Tissue Eng Part A 2010, 16:585-594.

9. Hsu YH, Moya ML, Abiri P, Hughes CC, George SC, Lee AP: Full range physiological mass transport control in 3D tissue cultures. Lab Chip 2013, 13:81-89.

10. Moya ML, Hsu YH, Lee AP, Hughes CC, George SC: In vitro perfused human capillary networks. Tissue Eng Part C Methods 2013, 19:730-737.

11. Singelyn JM, DeQuach JA, Seif-Naraghi SB, Littlefield RB, Schup-Magoffin PJ, Christman KL: Naturally derived myocardial matrix as an injectable scaffold for cardiac tissue engineering. Biomaterials 2009, 30:5409-5416.

12. Boudou T, Legant WR, Mu A, Borochin MA, Thavandiran N, Radisic M, Zandstra PW, Epstein JA, Margulies KB, Chen CS: A microfabricated platform to measure and manipulate the mechanics of engineered cardiac microtissues. Tissue Eng Part A 2012, 18:910-919.

13. Grosberg A, Alford PW, McCain ML, Parker KK: Ensembles of engineered cardiac tissues for physiological and pharmacological study: heart on a chip. Lab Chip 2011, 11:4165-4173.

14. Pegram M, Ngo D: Application and potential limitations of animal models utilized in the development of trastuzumab (Herceptin'): a case study. Adv Drug Deliver Rev 2006, 58:723-734.

15. Gai H, Leung EL-H, Costantino PD, Aguila JR, Nguyen DM, Fink LM, Ward DC, Ma Y: Generation and characterization of functional cardiomyocytes using induced pluripotent stem cells derived from human fibroblasts. Cell Biol Int 2009, 33:1184-1193.

16. Guo L, Abrams RMC, Babiarz JE, Cohen JD, Kameoka S, Sanders MJ, Chiao E, Kolaja KL: Estimating the risk of drug-induced proarrhythmia using human induced pluripotent stem cell-derived cardiomyocytes. Toxicol Sci 2011, 123:281-289.

17. Tulloch NL, Muskheli V, Razumova MV, Korte FS, Regnier M, Hauch KD, Pabon L, Reinecke H, Murry CE: Growth of engineered human myocardium with mechanical loading and vascular coculture. Circ Res 2011, 109:47-59.

18. Hansen A, Eder A, Bönstrup M, Flato M, Mewe M, Schaaf S, Aksehirlioglu BI, Schwoerer A, Uebeler J, Eschenhagen T: Development of a drug screening platform based on engineered heart tissue. Circ Res 2010, 107:35-44.

19. Zhang D, Shadrin IY, Lam J, Xian H-Q, Snodgrass HR, Bursac N: Tissueengineered cardiac patch for advanced functional maturation of human ESC-derived cardiomyocytes. Biomaterials 2013, 34:5813-5820.

20. Zhang J, Klos M, Wilson GF, Herman AM, Lian X, Raval KK, Barron MR, Hou L, Soerens $A G, Y u$ J: Extracellular matrix promotes highly efficient cardiac differentiation of human pluripotent stem cellsnovelty and significance. The matrix sandwich method. Circ Res 2012, 111:1125-1136.

21. Stringari $C$, Cinquin A, Cinquin O, Digman MA, Donovan PJ, Gratton E: Phasor approach to fluorescence lifetime microscopy distinguishes different metabolic states of germ cells in a live tissue. Proc Natl Acad Sci U S A 2011, 108:13582-13587.

22. Brantley-Sieders DM, Fang WB, Hicks DJ, Zhuang G, Shyr Y, Chen J: Impaired tumor microenvironment in EphA2-deficient mice inhibits tumor angiogenesis and metastatic progression. FASEB J 2005, 19:1884-1886.

23. Zhang Z, Ramirez NE, Yankeelov TE, Li Z, Ford LE, Qi Y, Pozzi A, Zutter MM: 
a2 $\beta 1$ integrin expression in the tumor microenvironment enhances tumor angiogenesis in a tumor cell-specific manner. Blood 2008, 111:1980-1988.

24. David L, Dulong V, Le Cerf D, Cazin L, Lamacz M, Vannier JP: Hyaluronan hydrogel: an appropriate three-dimensional model for evaluation of anticancer drug sensitivity. Acta Biomater 2008, 4:256-263.

25. Serebriiskii I, Castello-Cros R, Lamb A, Golemis EA, Cukierman E: Fibroblastderived $3 \mathrm{D}$ matrix differentially regulates the growth and drugresponsiveness of human cancer cells. Matrix Biol 2008, 27:573-585.

26. Weigelt B, Lo AT, Park CC, Gray JW, Bissell MJ: HER2 signaling pathway activation and response of breast cancer cells to HER2-targeting agents is dependent strongly on the 3D microenvironment. Breast Cancer Res Treat 2010, 122:35-43

27. Sutherland RM: Cell and environment interactions in tumor microregions the multicell spheroid model. Science 1988, 240:177-184.

28. Kunz-Schughart LA, Kreutz M, Knuechel R: Multicellular spheroids: a threedimensional in vitro culture system to study tumour biology. Int J Exp Pathol 1998, 79:1-23.

29. Correa de Sampaio PC, Auslaender D, Krubasik D, Failla AV, Skepper JN, Murphy G, English WR: A heterogeneous in vitro three dimensional model of tumour-stroma interactions regulating sprouting angiogenesis. Plos One 2012, 7: e30753.

30. Onozuka $\mathrm{H}$, Tsuchihara $\mathrm{K}$, Esumi $\mathrm{H}$ : Hypoglycemic/hypoxic condition in vitro mimicking the tumor microenvironment markedly reduced the efficacy of anticancer drugs. Cancer Sci 2011, 102:975-982.

31. Zhang C, Fu L, Fu J, Hu L, Yang H, Rong TH, Li Y, Liu H, Fu SB, Zeng YX, Guan $X Y$ : Fibroblast growth factor receptor 2-positive fibroblasts provide a suitable microenvironment for tumor development and progression in esophageal carcinoma. Clin Cancer Res 2009, 15:4017-4027.

32. Agarwal A, Coleno ML, Wallace VP, Wu WY, Sun CH, Tromberg BJ, George SC: Two-photon laser scanning microscopy of epithelial cell-modulated collagen density in engineered human lung tissue. Tissue Eng 2001, 7:191-202.

33. Ghajar CM, Blevins KS, Hughes CC, George SC, Putnam AJ: Mesenchymal stem cells enhance angiogenesis in mechanically viable prevascularized tissues via early matrix metalloproteinase upregulation. Tissue Eng 2006, 12:2875-2888.

34. Thompson HG, Mih JD, Krasieva TB, Tromberg BJ, George SC: Epithelialderived TGF- $\beta 2$ modulates basal and wound-healing subepithelial matrix homeostasis. Am J Physiol Lung Cell Mol Physiol 2006, 291:L1277-L1285.

35. Ghajar CM, Chen X, Harris JW, Suresh V, Hughes CC, Jeon NL, Putnam AJ, George SC: The effect of matrix density on the regulation of 3-D capillary morphogenesis. Biophys J 2008, 94:1930-1941.

36. Raub CB, Putnam AJ, Tromberg BJ, George SC: Predicting bulk mechanical properties of cellularized collagen gels using multiphoton microscopy. Acta Biomater 2010, 6:4657-4665.

doi: $10.1186 /$ scrt376

Cite this article as: Moya M, et al:: An integrated in vitro model of perfused tumor and cardiac tissue. Stem Cell Research \& Therapy 2013, 4(Suppl 1):S15. 